\title{
BOLOCAM OBSERVATIONS OF TWO UNCONFIRMED GALAXY CLUSTER CANDIDATES FROM THE PLANCK EARLY SUNYAEV-ZEL'DOVICH SAMPLE
}

\author{
J. Sayers ${ }^{1}$, N. G. Czakon ${ }^{1}$, C. Bridge ${ }^{1}$, S. R. Golwala ${ }^{1}$, P. M. Koch ${ }^{2}$, K.-Y. Lin ${ }^{2}$, S. M. Molnar ${ }^{3}$, And K. Umetsu Mnd $^{2}$ \\ ${ }^{1}$ Division of Physics, Math, and Astronomy, California Institute of Technology, Pasadena, CA 91125, USA; jack@ caltech.edu \\ ${ }^{2}$ Institute of Astronomy and Astrophysics, Academia Sinica, P.O. Box 23-141, Taipei 10617, Taiwan \\ ${ }^{3}$ LeCosPA Center, National Taiwan University, Taipei 10617, Taiwan \\ Received 2011 December 20; accepted 2012 March 6; published 2012 March 26
}

\begin{abstract}
We present Bolocam observations of two galaxy cluster candidates reported as unconfirmed in the Planck early Sunyaev-Zel'dovich (eSZ) sample, PLCKESZ G115.71+17.52 and PLCKESZ G189.84-37.24. We observed each of these candidates with Bolocam at $140 \mathrm{GHz}$ from the Caltech Submillimeter Observatory in 2011 October. The resulting images have white noise levels of $\simeq 30 \mu \mathrm{K}_{\mathrm{CMB}}$ arcmin in their central regions. We find a significant SZ decrement toward PLCKESZ G115.71. This decrement has a false detection probability of $5.3 \times 10^{-5}$, and we therefore confirm PLCKESZ G115.71 as a cluster. The maximum SZ decrement toward PLCKESZ G189.84 corresponds to a false detection probability of 0.027 , and it therefore remains as an unconfirmed cluster candidate. In order to make our SZ-derived results more robust, we have also analyzed data from the Wide-field Infrared Survey Explorer (WISE) at the location of each cluster candidate. We find an overdensity of WISE sources consistent with other clusters in the eSZ at the location of PLCKESZ G115.71, providing further evidence that it is a cluster. We do not find a significant overdensity of WISE sources at the location of PLCKESZ G189.84.
\end{abstract}

Key words: cosmology: observations - galaxies: clusters: individual (PLCKESZ G115.71, PLCKESZ G189.84)

Online-only material: color figures

\section{INTRODUCTION}

The thermal Sunyaev-Zel'dovich (SZ) effect describes the scattering of cosmic microwave background (CMB) photons with the hot electrons in the intracluster medium (ICM; Sunyaev \& Zel'dovich 1972). The surface brightness of the SZ effect is independent of redshift, and the integrated SZ signal is approximately equal to the total thermal energy of the ICM divided by the square of the angular diameter distance, making the SZ signal an excellent cluster mass proxy with little redshift evolution (Barbosa et al. 1996; Holder et al. 2000). Consequently, surveys using the SZ effect to detect clusters have an almost mass-limited selection function and can yield powerful constraints on cosmological parameters (Haiman et al. 2000; Holder et al. 2001). Three such SZ surveys are currently ongoing, conducted by the South Pole Telescope (SPT), the Atacama Cosmology Telescope, and the Planck satellite, and each has published initial cluster catalogs (Vanderlinde et al. 2010; Williamson et al. 2011; Marriage et al. 2011; Planck Collaboration 2011).

The Planck survey has released a list of 189 cluster candidates called the early SZ (eSZ) sample (Planck Collaboration 2011). Among this list, 169 are previously known clusters, and 12 were confirmed by the Planck team prior to the publication of the eSZ. Subsequent SZ observations by SPT and the Arcminute Microkelvin Imager (AMI) have confirmed five and one additional eSZ candidates, respectively (Story et al. 2011; Hurley-Walker et al. 2011). Prior to our original submission of this Letter, there had been no published confirmations of the remaining two cluster candidates in the eSZ sample, PLCKESZ G115.71+17.52 and PLCKESZ G189.84-37.24. ${ }^{4}$ We note that Planck

\footnotetext{
4 AMI observed, but did not detect, PLCKESZ G115.71 (Hurley-Walker et al. 2011). Furthermore, Muchovej et al. (2012) published an SZA detection of PLCKESZ G115.71, and an SZA non-detection of PLCKESZ G189.84, after our original submission of this Letter.
}

Collaboration (2011) lists possible contamination from dust emission near PLCKESZ G115.71 and a high level of contamination from Galactic emission toward G189.84. Additionally, Planck Collaboration (2011) specifically notes that G189.84 is a low reliability source, and is the only unconfirmed eSZ candidate without a ROSAT All-Sky Survey (RASS) association.

\section{OBSERVATIONS}

Bolocam is a millimeter-wave imaging camera that operates from the Caltech Submillimeter Observatory with 144 bolometric detectors covering a circular 8 arcmin field of view (FOV) with $\simeq 1$ arcmin resolution at the SZ-emission-weighted band center of $140 \mathrm{GHz}$ used for these observations (Glenn et al. 1998; Haig et al. 2004). The data described in this Letter were obtained by scanning the telescope in a Lissajous pattern to yield maps with tapered coverage out to $\simeq 10$ arcmin in radius centered on the Planck eSZ coordinates (identical to the observations described in Sayers et al. (2011)). The observations were conducted in 2011 October, almost entirely in very poor weather conditions with a $225 \mathrm{GHz}$ opacity of $\tau_{225} \simeq 0.20$. The total integration time was $12 \mathrm{hr}$ for PLCKESZ G115.71 and $7.5 \mathrm{hr}$ for PLCKESZ G189.84, yielding images with central noise rms of $29.5 \mu \mathrm{K}_{\mathrm{CMB}}$ arcmin and $36.5 \mu \mathrm{K}_{\mathrm{CMB}}$ arcmin. Note that SPT reached rms of $28-39 \mu \mathrm{K}_{\mathrm{CMB}}$ arcmin in their targeted observations of eSZ clusters, which resulted in detections with peak signal-to-noise ratios $(\mathrm{S} / \mathrm{Ns}$ ) ranging from 6.3 to 13.8 (Story et al. 2011).

\section{SZ DATA ANALYSIS}

These data were reduced according to the procedures described in detail in Sayers et al. (2011), with only minor changes. We briefly summarize the data reduction here. First, we used frequent observations of bright quasars to obtain pointing corrections accurate to 5 arcsec, and we used observations of Uranus 
Table 1

Bolocam SZ Observation Details

\begin{tabular}{lcccccc}
\hline \hline $\begin{array}{l}\text { Name } \\
\text { PLCKESZ }\end{array}$ & $\begin{array}{c}\text { Noise rms } \\
\left(\mu \mathrm{K}_{\mathrm{CMB}} \operatorname{arcmin}\right)\end{array}$ & $\begin{array}{c}\mathrm{S} / \mathrm{N} \\
\zeta\end{array}$ & $\begin{array}{c}\text { R.A. } \\
(\mathrm{J} 2000)\end{array}$ & $\begin{array}{c}\Delta \text { R.A. } \\
(\operatorname{arcsec})\end{array}$ & $\begin{array}{c}\text { Decl. } \\
(\mathrm{J} 2000)\end{array}$ & $\begin{array}{c}\Delta \text { Decl. } \\
(\operatorname{arcsec})\end{array}$ \\
\hline G115.71 & 29.5 & 5.7 & $22: 26: 27.3$ & +0.1 & $+78: 19: 15$ & +29.7 \\
G189.84 & 36.5 & 3.8 & $03: 59: 46.5$ & +10.4 & $+00: 06: 34$ & +25.8 \\
\hline
\end{tabular}

Notes. From left to right the columns give the name, the Bolocam noise rms, the peak $\mathrm{S} / \mathrm{N} \zeta$, the Bolocam R.A./decl. centroid for that peak, and corresponding offset of this peak from the Planck coordinates.

and Neptune to obtain flux calibration accurate to $5 \%$. In particular, the flux calibration has been updated based on recent Wilkinson Microwave Anisotropy Probe results as described in detail in Sayers et al. (2012). The FOV-average signal is then subtracted at each time sample and the data time streams are high-pass filtered at $250 \mathrm{mHz}$ in order to remove atmospheric brightness fluctuations. Consequently, the cluster images are high-pass filtered in a way which we characterize via simulation to obtain a signal transfer function for each cluster.

We searched for clusters in these images using a matched filter according to the formalism described in Vanderlinde et al. (2010) (see also Haehnelt \& Tegmark 1996; Herranz et al. 2002a, 2002b; Melin et al. 2006; Marriage et al. 2011). We approximate the cluster SZ surface brightness profile $S(\theta)$ as

$$
S(\theta) \propto\left(1+\theta^{2} / \theta_{c}^{2}\right)^{-1}
$$

where $\theta$ is an angular distance and $\theta_{c}$ is the core radius. We then form a filter according to

$$
\psi(\theta)=\mathcal{F} \mathcal{T}^{-1}\left(\frac{\tilde{W}(u) \tilde{S}(u) \tilde{B}(u)}{\tilde{N}(u)}\right),
$$

where $\tilde{W}(u)$ is the signal transfer function in Fourier space, $\tilde{S}(u)$ is the cluster profile in Fourier space, $\tilde{B}(u)$ is the point-spread function (PSF) in Fourier space, $\tilde{N}(u)$ is the map-noise power spectrum, $u$ is an angular frequency, and $\mathcal{F} \mathcal{T}^{-1}$ denotes the Fourier transform back to map space. We filter our images in map space by convolving them with $\psi(\theta)$, after truncating $\psi(\theta)$ at a radius of $4 \mathrm{arcmin}$. This truncation is required to prevent the filter from introducing noise in the central region of the images from the low-coverage outer regions.

Analogous to Vanderlinde et al. (2010), each image is filtered using 12 different filters, with $\theta_{c}$ varied from 0.25 to 3.00 arcmin in 0.25 arcmin increments (note that our PSF has an FWHM of $\simeq 1$ arcmin), and we define our detection significance $\zeta$ as the peak $\mathrm{S} / \mathrm{N}$ found in any of these filtered images within an aperture of 4 arcmin radius centered on the Planck eSZ coordinates. This search radius was chosen because it corresponds to the upper limit on the Planck astrometry for all but the lowest redshift clusters (Planck Collaboration 2011). We find $\zeta=5.7$ for PLCKESZ G115.71 (with $\theta_{c}=0.25$ ) and $\zeta=3.8$ for PLCKESZ G189.84 (with $\theta_{c}=0.25$ ). The centroids of these maxima are given in Table 1 , and each is within 1 arcmin of the coordinates given in the Planck eSZ. Figure 1 shows thumbnails of each target filtered with the filter that produces the maximum $\mathrm{S} / \mathrm{N}$.

We characterize our detection significance via simulation by generating noise maps from the combination of jackknife realizations of our data and a model for the astronomical fluctuations due to the $\mathrm{CMB}$ and point sources based on SPT measurements of the millimeter-wave power spectrum
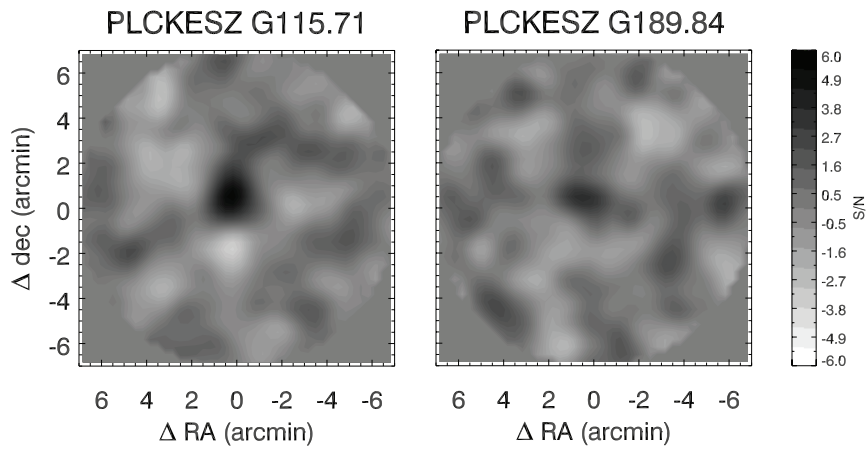

Figure 1. Signal-to-noise thumbnails of the SZ data centered on the Planck eSZ coordinates. Each thumbnail has been filtered with the filter that produces the maximum peak $\mathrm{S} / \mathrm{N} \zeta$ (equal to 5.7 for PLCKESZ G115.71 and equal to 3.8 for PLCKESZ G189.84).

(A color version of this figure is available in the online journal.)

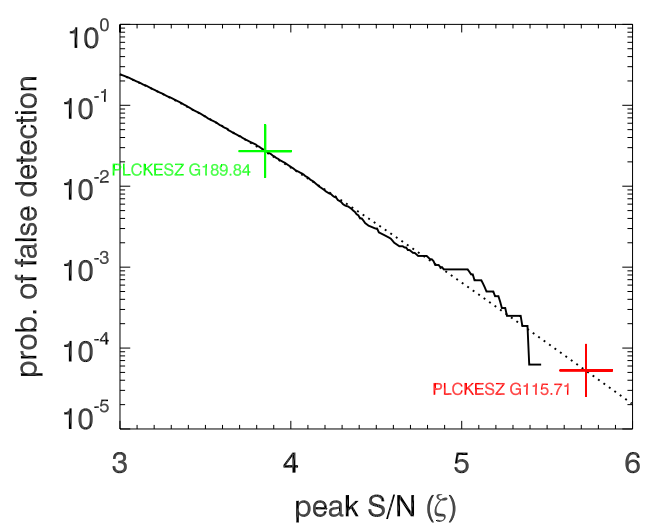

Figure 2. Probability of a false detection within our 4 arcmin search radius as a function of peak $\mathrm{S} / \mathrm{N} \zeta$. The solid line shows the results from simulations using 16,000 noise realizations and the dotted line is the best-fit model to these results. The values of $\zeta$ for each cluster candidate are shown as labeled crosses. Note that, compared to the similar plot shown in Figure 2 of Vanderlinde et al. (2010), our false detection probability at a given value of $\zeta$ is approximately three times larger compared to SPT. The likely causes for this difference are described in Section 3.

(A color version of this figure is available in the online journal.)

(Sayers et al. 2011; Reichardt et al. 2011). We generated 1000 such realizations for each target, along with the 14 other targets observed with Bolocam in 2011 October to similar depths. We then searched for the peak $\mathrm{S} / \mathrm{N}$ in each of these noise realizations, the distributions of which were statistically identical for all 16 targets. We therefore used all 16,000 measurements to characterize the probability of measuring a given peak $\mathrm{S} / \mathrm{N}$, with the results shown in Figure 2. We find the probability of a false detection of PLCKESZ G115.71 is $5.3 \times 10^{-5}$, and we therefore confirm it as a cluster. Note that PLCKESZ G115.71 has $\zeta=5.7$, which exceeds the value of $\zeta$ obtained in all 16,000 of our noise realizations, we have therefore extrapolated our simulation results using a parametric fit. The probability of a false detection of PLCKESZ G189.84 is 0.027 , and we therefore do not claim a detection of this candidate.

At a given value of $\zeta$, our false detection rate per given map area is approximately three times higher than the SPT false detection rate described in Vanderlinde et al. (2010). The false detection probability is similar when $\zeta$ (Bolocam) $\simeq$ $\zeta(\mathrm{SPT})+0.3$. Although SPT survey data are similar to our Bolocam data in many respects, there are subtle differences that contribute to this increase in our false detection probability. 

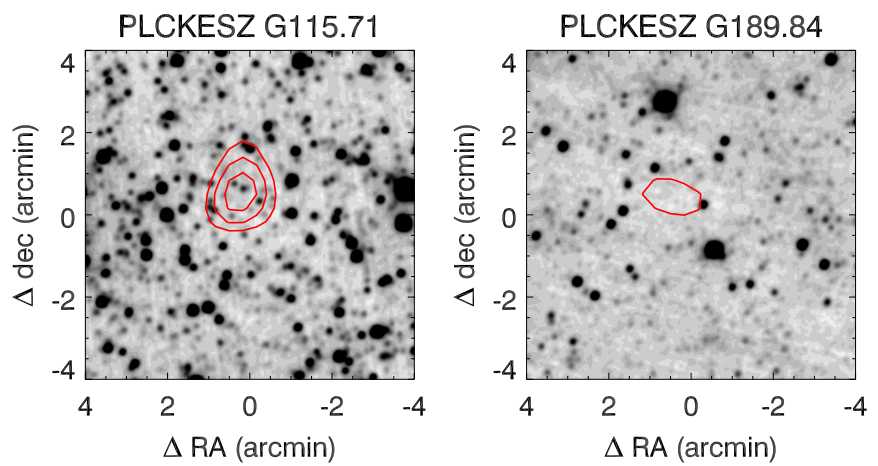

Figure 3. WISE band W1 thumbnails centered on the Planck eSZ coordinates of each eSZ candidate. Overlaid in red is the Bolocam SZ S/N with a contour spacing of 1 starting with an $\mathrm{S} / \mathrm{N}$ of -3 .

(A color version of this figure is available in the online journal.)

In particular, the Bolocam images have tapered coverage and consequently non-uniform noise properties. This means that for a given map pixel our filter will introduce noise from both lower- and higher-coverage regions of the map, and the filter is therefore not as optimal as the filter used by SPT.

\section{WISE DATA ANALYSIS}

We have also analyzed the Wide-field Infrared Survey Explorer (WISE) public-release data (Wright et al. 2010) at the location of each eSZ candidate. The central wavelengths of the WISE bands, which are at 3.4, 4.6, 12, and $22 \mu \mathrm{m}$, are too long to search for these candidates using a red-sequence technique (Gladders \& Yee 2000), and so we instead looked for overdensities of sources in the WISE images. Specifically, we counted the total number of sources in the public-release WISE catalogs for band $\mathrm{W} 1(3.4 \mu \mathrm{m})$ within a 3 arcmin radius aperture centered on our Bolocam SZ centroid. This aperture size is motivated by the fact that it corresponds to $\simeq 1 \mathrm{Mpc}$ at $z=0.4$, the median redshift of the six clusters originally published as unconfirmed in the eSZ (Story et al. 2011; Hurley-Walker et al. 2011). These source counts were then compared to counts within 1000 randomly located and identically sized apertures in the region within $\simeq 2^{\circ}$ of each eSZ candidate. We limit these blank-sky apertures to the region immediately surrounding the eSZ candidates because there are variations in WISE coverage and in the density of nearby sources, which causes the blank-sky source density to vary over the WISE survey. We find 140 sources within 3 arcmin of PLCKESZ G115.71 compared to a local-blank-skyaverage of 108 sources, and 9/1000 nearby blank-sky regions have more than 140 sources. We find 67 sources within 3 arcmin of PLCKESZ G189.84 compared to a local-blank-sky-average of 57 sources, and 168/1000 nearby blank-sky regions have more than 67 sources (see Figures 3 and 4).

\section{DISCUSSION}

In addition to the two eSZ candidates described in this Letter, we have also observed 12 other eSZ clusters as part of separate Bolocam programs (Abell 209, Abell 697, Abell 963, Abell 2204, Abell 2219, Abell 2261, Abell S1063, MACS J0417.5, MACS J0717.5, MACS J1149.5, MACS J1206.2, and MACS J2211.7). Results for Abell 697 have already been published in Sayers et al. (2011), and results for the other clusters will appear in future publications, including a detailed comparison of the Bolocam and Planck measurements. The Planck eSZ candidates were selected based on the millimeter/submillimeter-wave SZ signal strength measured by Planck, and the full eSZ catalog spans a relatively narrow range of SZ signal strengths. ${ }^{5}$ Since Bolocam is sensitive to the millimeter-wave SZ signal from these clusters, we therefore expect a correspondingly narrow spread in the SZ signals measured by Bolocam. However, we note that the vastly different angular scales probed by Planck $(\theta \geqslant 5$ arcmin) and Bolocam ( $1 \leqslant \theta \leqslant 15$ arcmin) may weaken the correlation between SZ measurements from Planck and Bolocam.

Since the Bolocam observations of these previously known eSZ clusters span a range of noise rms, from 16 to $49 \mu \mathrm{K}_{\mathrm{CMB}}$ arcmin, with peak $\mathrm{S} / \mathrm{Ns} \zeta=8.3-21.9$, we scale the Bolocam values of $\zeta$ to the expected value of $\zeta$ at a noise rms of $30 \mu \mathrm{K}_{\mathrm{CMB}}$ arcmin $\left(\zeta_{30}\right)$ in order to estimate the absolute SZ signal measured by Bolocam. ${ }^{6}$ As expected, we find that the values of $\zeta_{30}$ have a relatively narrow spread, ranging from 10.3 to 20.2 with a median value of $\simeq 14$ (with the exception of Abell 2261, which has $\zeta_{30}=5.4$ ). Consequently, our detection of PLCKESZ G115.71, with $\zeta=5.7$ and a noise rms of $29.5 \mu \mathrm{K}_{\mathrm{CMB}}$ arcmin, indicates that the SZ signal from this cluster is $\simeq 2.5$ times weaker than the median SZ signal

\footnotetext{
$5 \simeq 2 / 3$ of the eSZ candidates have Planck $\mathrm{S} / \mathrm{N}$ between 6 and 10 and $\simeq 95 \%$ of the eSZ candidates have Planck S/N between 6 and 15. In particular, the 12 previously known eSZ clusters observed with Bolocam have Planck S/Ns of 6.8-17.4.

6 As explained in Williamson et al. (2011), the SPT peak S/N does not scale linearly with noise rms due to changes in the map-noise spectrum and optimal filter shape resulting from changes in the effective noise ratios of the primary $\mathrm{CMB}$ and the instrument/atmospheric noise. In contrast, the more severe high-pass filtering applied to our data significantly reduces the amount of primary CMB noise in our images, and our map-noise spectrum and filter shape are approximately independent of noise rms.
}
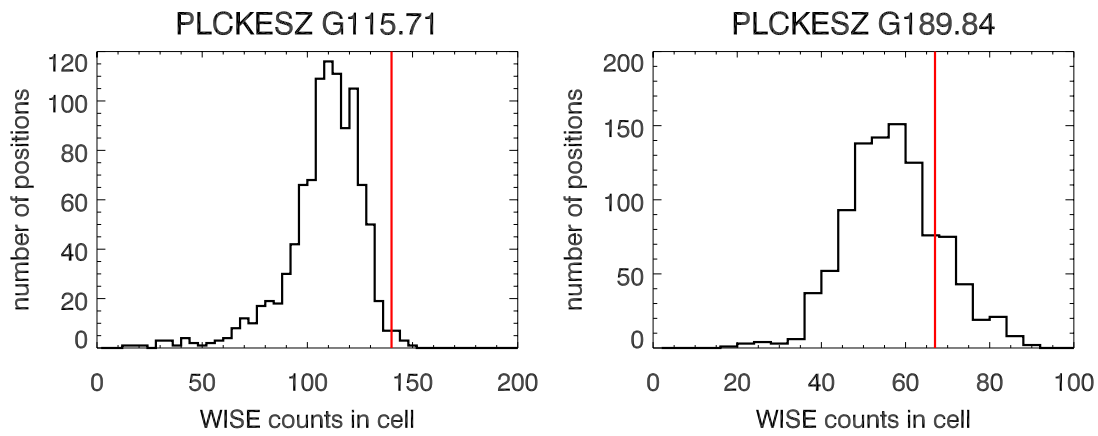

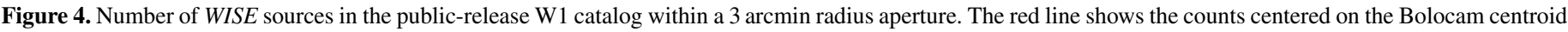

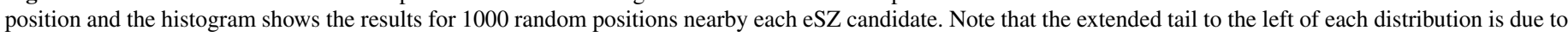
the WISE bright source masking algorithm.

(A color version of this figure is available in the online journal.) 
from Bolocam observations of other eSZ clusters (although not quite as weak as the signal from Abell 2261). Similarly, our image of PLCKESZ G189.84, which does not contain a significant detection and has a value of $\zeta=3.8$ and a noise rms of $36.5 \mu \mathrm{K}_{\mathrm{CMB}}$ arcmin, indicates that any $\mathrm{SZ}$ signal from that location is at least a factor of $\simeq 3$ times weaker than the median eSZ cluster. We discuss our results for each candidate in more detail below.

\subsection{PLCKESZ115.71}

One possible explanation for the weaker-than-expected SZ signal from PLCKESZ G115.71 is the existence of positive flux immediately south of the eSZ coordinates, which appears to be unresolved (see Figure 1). This unresolved positive flux has an $\mathrm{S} / \mathrm{N} \simeq 4$. We note that there is a source in the $1.4 \mathrm{GHz}$ NVSS catalog at 22:26:37.88, +78:17:17.6 (J2000), which closely matches the location of the positive flux in our image (Condon et al. 1998). However, two nearby sources detected by AMI at $15 \mathrm{GHz}$ have flux densities of $\simeq 1 \mathrm{mJy}$, indicating that the radio component of the NVSS source has a sharply falling spectrum and should not be detected in our $140 \mathrm{GHz}$ image (HurleyWalker et al. 2011). In addition, the Planck Collaboration notes possible contamination from dust emission for this candidate (Planck Collaboration 2011). However, due to the low detection significance of this positive flux in our image, and our lack of multi-band data, it is not clear if this positive flux is due to astronomical sources, noise, or both. If this positive flux in our image of PLCKESZ G115.71 is due to astronomical sources, then our estimate of both the centroid and the strength of the SZ signal from PLCKESZ G115.71 may be biased, and it may explain why we measure a weaker-than-expected SZ signal.

Of the 12 additional eSZ clusters observed by Bolocam, 6 are contained in the currently available public WISE data (Abell 2204, Abell 2219, Abell 2261, Abell 697, MACS J0417.5, and MACS J0717.5). We counted the number of W1 sources within a 3 arcmin radius of each of these clusters, and again compared those results to the counts in 1000 nearby background regions. We find that the median number of nearby regions with more WISE sources than the cluster locations is 6/1000 for these clusters. Our results for PLCKESZ G115.71 (with more WISE sources than 9/1000 nearby regions) are therefore consistent with WISE data for previously known eSZ clusters.

\subsection{PLCKESZ G189.84}

Our Bolocam observations toward PLCKESZ G189.84 do not contain a conclusive detection of an SZ signal; while there are indications of a decrement, the false detection probability is 0.027 , which is too large to claim a detection. Additionally, while WISE data show a slight overdensity of objects in the direction of this candidate, the overdensity is consistent with a statistical fluctuation (17\% probability to exceed). Given the lack of a conclusive detection at a signal level similar to other sources in the eSZ catalog there are (at least) three possible explanations for the observed results: (1) There is a cluster with properties that are unique compared to others in the eSZ catalog (weak SZ signal, weak WISE signal, and no RASS association). (2) The Planck detection, along with the weak signals seen in the Bolocam and WISE data, are statistical fluctuations, though this seems unlikely given the expected purity of the eSZ catalog combined with the fact that both the Bolocam and WISE excursions are in the direction of consistency with the presence of a cluster. (3) Millimeter/submillimeter-wave astrophysical contaminants produced the Planck detection and caused the nonsignificant excursion seen in the Bolocam data; this appears to be a reasonable explanation, especially given the high level of galactic contamination noted by Planck Collaboration (2011). A definitive conclusion about PLCKESZ G189.84 will require additional data, in particular results from the full-depth Planck survey (along with the inclusion of data from the Planck LFI).

\section{CONCLUSIONS}

In 2011 October we performed dedicated $140 \mathrm{GHz}$ Bolocam observations of two cluster candidates from the Planck eSZ sample, PLCKESZ G115.71 and PLCKESZ G189.84. These observations total $\simeq 10 \mathrm{hr}$ each, and produce images with a noise rms of $\simeq 30 \mu \mathrm{K}_{\mathrm{CMB}}$ arcmin. From these images we have confirmed PLCKESZ G115.71 as a cluster at high significance, with a false detection probability of $5.3 \times 10^{-5}$. We are not able to confirm PLCKESZ G189.84 as a cluster, although our image does show a weak decrement with a false detection probability of 0.027. Both our detection of PLCKESZ G115.71 and our non-detection of PLCKESZ G189.84 indicate SZ signals significantly lower than expected based on Bolocam observations of other eSZ cluster candidates which are known to be true clusters. However, we note the presence of positive flux immediately south of the SZ decrement for PLCKESZ G115.71, which may be partially filling in the SZ decrement from the cluster. We have also examined WISE data at the positions of these cluster candidates, and find a significant overdensity of sources toward PLCKESZ G115.71, providing further evidence that it is a cluster.

We acknowledge the assistance of: the day crew and Hilo staff of the Caltech Submillimeter Observatory, who provided invaluable assistance during data taking for this data set; Kathy Deniston, Barbara Wertz, and Diana Bisel, who provided effective administrative support at Caltech and in Hilo; and the referees of our Letter, who provided many useful comments. The Bolocam observations were supported by the Gordon and Betty Moore Foundation. J.S. was supported by NSF/AST-0838261 and NASA/NNX11AB07G; N.C. was partially supported by a NASA Graduate Student Research Fellowship; K.U. acknowledges support from the Academia Sinica Career Development Award. This publication makes use of data products from the Wide-field Infrared Survey Explorer, which is a joint project of the University of California, Los Angeles, and the Jet Propulsion Laboratory/California Institute of Technology, funded by the National Aeronautics and Space Administration.

\section{REFERENCES}

Barbosa, D., Bartlett, J. G., Blanchard, A., \& Ouikbir, J. 1996, A\&A, 314, 13 Condon, J. J., Cotton, W. D., Greisen, E. W., et al. 1998, AJ, 115, 1963 Gladders, M. D., \& Yee, H. K. C. 2000, AJ, 120, 2148

Glenn, J., Bock, J. J., Chattopadhyay, G., et al. 1998, Proc. SPIE, 3357, 326 Haehnelt, M. G., \& Tegmark, M. 1996, MNRAS, 279, 545

Haig, D. J., Ade, P. A. R., Mauskopf, P. D., et al. 2004, Proc. SPIE, 5498, 78 Haiman, Z., Mohr, J. J., \& Holder, G. P. 2000, ApJ, 553, 545

Herranz, D., Sanz, J., Barreiro, R. B., \& Martínez-Gonález, E. 2002a, ApJ, 580, 610

Herranz, D., Sanz, J. L., Hobson, M. P., et al. 2002b, MNRAS, 336, 1057

Holder, G. P., Haiman, Z., \& Mohr, J. J. 2001, ApJ, 560, L111

Holder, G. P., Mohr, J. J., Carlstrom, J. E., Evrard, A. E., \& Leitch, E. M. 2000, ApJ, 544, 629

Hurley-Walker, N., Brown, M. L., Davies, M. L., et al. 2011, MNRAS, 414, L75

Marriage, T. A., Acquaviva, V., Ade, P. A. R., et al. 2011, ApJ, 737, 61

Melin, J.-B., Bartlett, J. G., \& Delabrouille, J. 2006, A\&A, 459, 341 
Muchovej, S., Leitch, E., Culverhouse, T., Carpenter, J., \& Sievers, J. 2012 arXiv: 1202.0527

Planck Collaboration, Ade, P. A. R., Aghanim, N., et al. 2011, A\&A, 536, A8

Reichardt, C. L., Shaw, L., Zahn, O., et al. 2011, arXiv:1111.0932

Sayers, J., Czakon, N. G., \& Golwala, S. R. 2012, ApJ, 744, 169

Sayers, J., Golwala, S. R., Ameglio, S., \& Pierpaoli, E. 2011, ApJ, 728, 39
Story, K., Aird, K. A., Andersson, K., et al. 2011, ApJ, 735, L36

Sunyaev, R. A., \& Zel'dovich, Y. B. 1972, Comments Astrophys. Space Phys., 4,173

Vanderlinde, K., Crawford, T. M., de Haan, T., et al. 2010, ApJ, 722, 1180

Williamson, R., Benson, B. A., High, F. W., et al. 2011, ApJ, 738, 139

Wright, E. L., Eisenhardt, P. R. M., Mainzer, A. K., et al. 2010, AJ, 140, 1868 